\title{
Desenvolvimento e caracterização físico-química de geleia comum e extra de graviola com pimenta
}

\author{
Ana Claudia Martins Teles ${ }^{1}$, Ellen Godinho Pinto ${ }^{1}$, Janyne Ribeiro dos Santos ${ }^{1}$, Camila \\ Fernanda Dias de Oliveira ${ }^{1}$, Dayana Silva Batista Soares ${ }^{1}$ \\ ${ }^{1}$ Instituto Federal Goiano - IF Goiano, Campus Morrinhos, Goiás, Brasil. E-mail: acmstsprmtdlms@ @otmail.com, \\ ellengodinho@hotmail.com, janine.ribeiro@hotmail.com, camilaferdias@gmail.com, dayana.soares@ifgoiano.edu.br
}

Recebido: 07/11/2016; Aceito: 01/03/2017.

\section{RESUMO}

A produção de geleias é um processo bastante utilizado, porém algumas frutas com grande quantidade de nutrientes, como é o caso da graviola, são ainda pouco exploradas comercialmente nesses processos. Neste trabalho teve-se como objetivo o desenvolvimento e caracterização de geleia de graviola (Annona muricata) com pimenta "dedo de moça" tipo extra e comum em três concentrações de pectina 0,$5 ; 1,0$ e 1,5\%. As análises físicas e químicas foram analisadas quanto ao seu teor de sólidos solúveis totais, umidade, vitamina C, Aw (atividade de água), acidez e $\mathrm{pH}$, teste de sinérese e coloração expressa com valores de $\mathrm{L}^{*}, \mathrm{a}^{*}, \mathrm{~b}^{*}$, croma e hue. A acidez e umidade foram influenciadas pela concentração de pectina e também pela concentração de açúcar na geleia extra e comum. Apenas a geleia comum com $0,5 \%$ de pectina apresentou sinérese, devido a maior acidez. Houve diferença significativa entre a geleia extra e comum na umidade e acidez e Aw e entre os parâmetros de cor na geleia extra e comum. Resultados demonstraram que a estrutura do gel é afetada pela concentração do açúcar, pectina e pela acidez.

Palavras-chave: pectina, sinérese, croma e hue.

\section{Development and physico-chemical characterization of common and extra jelly soursop with pepper}

\begin{abstract}
The production of gel is a widely-used process, but some fruits with lot of nutrients, such as the soursop, are still not commercially exploited in these processes. This work had as objective the development and characterization of soursop fruit (Annona muricata) jelly with pepper "lady finger" and extra common type in three pectin concentrations $0.5 ; 1.0$ and $1.5 \%$. The physical and chemical analyzes were analyzed for total soluble solids content, moisture, vitamin $\mathrm{C}$, Aw (water activity), acidity and $\mathrm{pH}$, syneresis test and expressed staining L *, a *, b * chroma and hue. The acidity and humidity were influenced by the concentration of pectin and, also the concentration of sugar in the extra common and jelly. Only the common jelly with $0.5 \%$ pectin presented syneresis due to higher acidity. There was a significant difference between the extra and common jam in moisture and acidity, Aw and between the color parameters in common and extra jelly. Results showed that the gel structure is affected by the concentration of sugar, pectin and by the acidity.
\end{abstract}

Key words: pectin, syneresis, chroma and hue. 


\section{Introdução}

A graviola (Annona muricata), pertencente à família Annonaceae, é uma das importantes frutíferas cultivadas no Nordeste Brasileiro, principalmente nos Estados da Paraíba, Ceará, Pernambuco e Bahia; seus frutos utilizados na fabricação de suco, sorvetes, compotas, geleias e doces (SACRAMENTO, 2003).

As pimentas do gênero Capsicum são originadas do continente americano e pertencem à família das Solanáceas, e dentre as espécies deste gênero encontramos a Capsicum baccatum (dedo-de-moça) que apresenta frutos de cores e formas variadas, cultivada originariamente no sudeste e sul do Brasil (CARVALHO; BIANCHETTI, 2004).

Segundo o Instituto Adolf Lutz (2008), geleias de frutas são produtos preparados a partir de frutas e/ou sucos, misturados com açúcar, com adição de pectina, ácidos e outros ingredientes permitidos, podendo apresentar frutas inteiras, partes e/ou pedaços sob variadas formas, formando uma mistura que será processada até se obter uma concentração e consistência semi-sólidas adequada. A Legislação permite a adição de acidulantes e de pectina para compensar qualquer deficiência no conteúdo natural de pectina ou acidez da fruta (TORREZAN, 1998).

Neste trabalho objetivou-se desenvolver formulações de geleia de graviola (Annona muricata) do tipo comum e extra com pimenta "dedo de moça" (Capsicum baccatum) elaboradas com diferentes concentrações de pectina avaliando possíveis influências nas propriedades físicas e químicas entre os dois tipos de geleia, a comum e a extra.

\section{Material e Métodos}

As formulações de geleia, comum e extra, foram desenvolvidas no Instituto Federal Goiano - Campus Morrinhos, no laboratório de Agroindústria, onde foram conduzidas as análises físicas e químicas na geleia de graviola com pimenta tipo extra e comum.

\section{Obtenção das geleias}

As Tabelas 1 e 2 apresentam a formulação de cada geleia de graviola com pimenta comum e extra, respectivamente.

As formulações foram produzidas de acordo com Pereira et al. (2011), com adaptações. Na Figura 1 apresenta o fluxograma da geleia comum e extra de graviola com pimenta.

Após sanitização, foi realizado o descascamento, corte e retirada manual das sementes, separação dos gomos e adição da polpa em tacho de cobre juntamente com o açúcar na proporção de 40 partes de polpa da fruta para 60 partes de açúcar, para geleia do tipo comum e 50 partes de polpa para 50 de açúcar na geleia extra, conforme estabelecido pela Resolução CNNPA $\mathrm{n}^{\circ} 12$, de 1978 (BRASIL, 1978).

Foi adicionado ácido cítrico e pectina concentrado até atingir o ${ }^{\circ}$ Brix desejado. As formulações obtidas foram envasadas e acondicionadas (Figura 2) até o momento das análises, em embalagens de vidro de 200 $\mathrm{g}$, previamente esterilizadas, fechadas com tampa de metal e imediatamente resfriadas em água fria.

Tabela 1. Porcentagem dos ingredientes utilizados nas formulações de geleia comum de graviola com adição de pimenta nos teores de pectina $0,5 \%, 1,0 \%$, e $1,5 \%$.

\begin{tabular}{|c|c|c|c|}
\hline Matérias-primas & $\mathrm{F} 1(0,5 \%)$ & $\mathrm{F} 2(1,0 \%)$ & F3(1,5\%)* \\
\hline Açúcar & 64,44 & 64,33 & 64,20 \\
\hline Graviola & 32,20 & 32,15 & 32,12 \\
\hline Pimenta & 3,20 & 3,20 & 3,20 \\
\hline Pectina & 0,16 & 0,32 & 0,48 \\
\hline
\end{tabular}

*Porcentagem de pectina equivalente a 0,5\% (F1), 1,0\% (F2), 1,5\% (F3) de 100\% do peso do açúcar cristal.

Tabela 2. Porcentagem dos ingredientes utilizados nas formulações de geleia extra de graviola com adição de pimenta nos teores de pectina $0,5 \%, 1,0 \%$, e $1.5 \%$.

\begin{tabular}{|c|c|c|c|}
\hline Matérias-primas & $\mathrm{F} 1(0,5 \%)$ & $\mathrm{F} 2(1,0 \%)$ & F3(1,5\%)* \\
\hline Açúcar & 48,44 & 48,33 & 48,20 \\
\hline Graviola & 48,44 & 48,33 & 48,20 \\
\hline Pimenta & 3,20 & 3,20 & 3,20 \\
\hline Pectina & 0,16 & 0,32 & 0,48 \\
\hline
\end{tabular}

*Porcentagem de pectina equivalente a $0,5 \%(\mathrm{~F} 1), 1,0 \%$ (F2), 1,5\% (F3) de $100 \%$ do peso do açúcar cristal. 


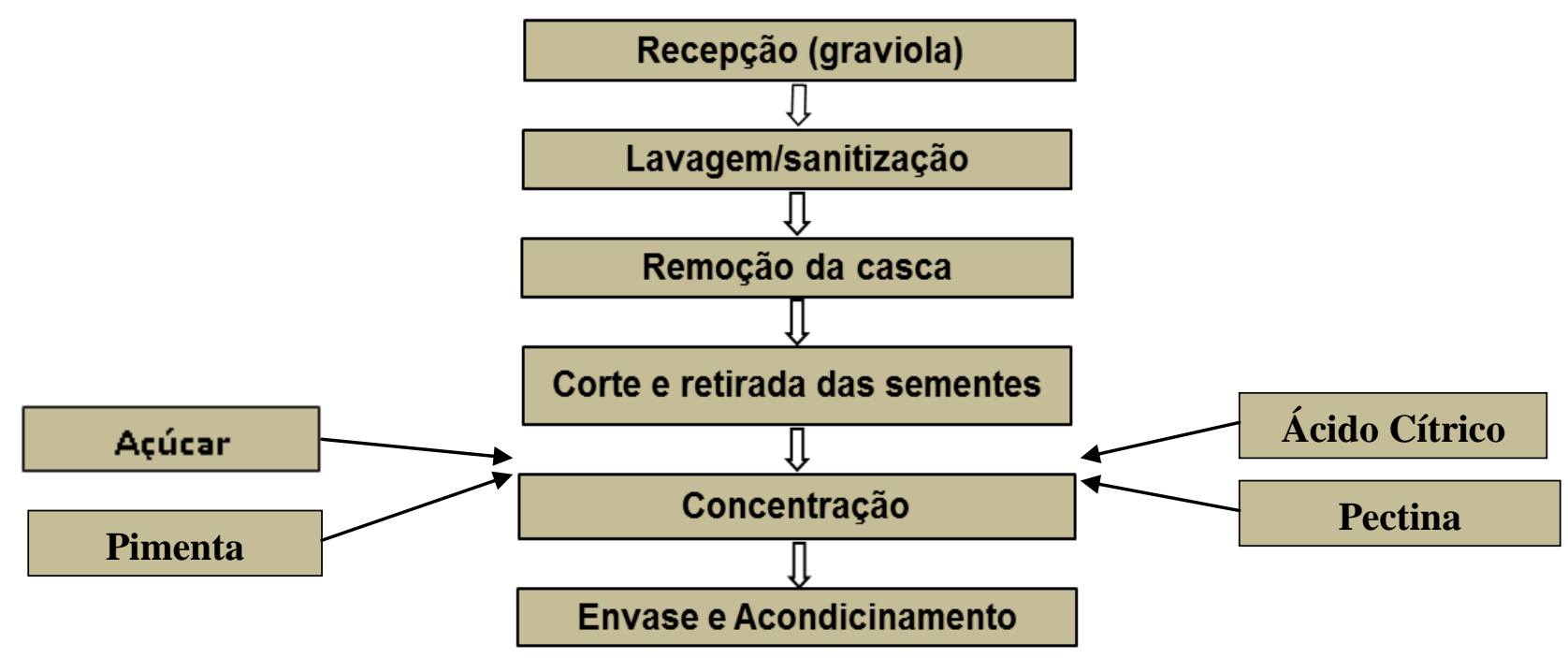

Figura 1. Fluxograma das formulações de geleia (comum e extra) de graviola (Annona muricata) com pimenta.

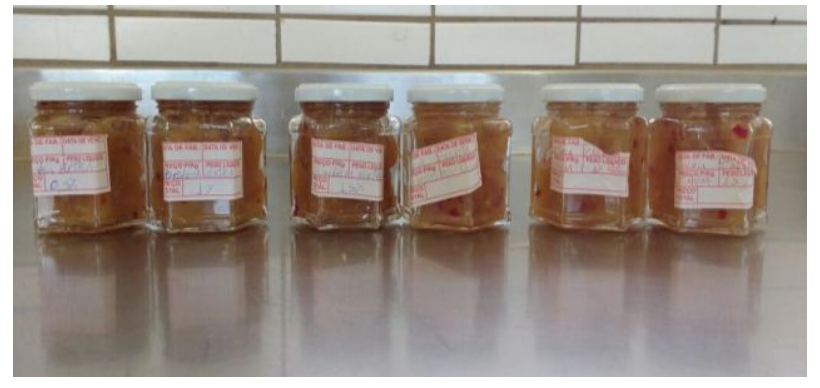

Figura 2. Acondicionamento das Formulações de geleia de graviola com pimenta.

\section{Análises Químicas e Físicas}

As geleias foram submetidas às análises de sólidos solúveis totais ( $\left.{ }^{\circ} \mathrm{Brix}\right), \mathrm{pH}$, acidez titulável, vitamina $\mathrm{C}$, umidade. As análises foram realizadas segundo metodologia do Instituto Adolf Lutz (2008) para as características cor e atividade de água (Aw); para análise do equipamento e da sinérese foi utilizada metodologia de acordo com Licodiedolff et al. (2010).

O procedimento para análise de sinérese foi feito segundo Licodiedolff et al. (2010). A presença de sinérese na geleia foi determinada por gravimetria utilizando-se um béquer de $250 \mathrm{~mL}$ e uma peneira comum. O volume de líquido depositado no fundo do béquer foi pesado e utilizado para o cálculo da porcentagem de sinérese de acordo com a Equação 1. Os resultados foram expressos em grama de líquido liberado/100g de produto.

$$
\text { Sinerese }(\%)=\frac{\text { gdelíquido }}{100 \mathrm{~g}}
$$

A determinação de cor foi realizada utilizando-se colorímetro Minolta, devidamente calibrado. Resultados foram expressos em "CIELAB" (CIE L*a*b*), espaço de cor comumente utilizado para determinações de cor em alimentos.

A partir desses dados foram calculados os valores de croma $\left(\mathrm{c}^{*}\right)$, que correspondem à saturação ou intensidade da cor (Eq. 2):

$$
c^{*}=\sqrt{a^{2}+b^{2}}
$$

Foram calculados tambe os valores de ângulo de tonalidade (ângulo $h^{\circ}$ ), expressa em graus, pela Eq. 3:

$$
\left.\mathrm{h}^{*}=\operatorname{tang}^{-1(} \mathrm{b} * / \mathrm{a}^{*}\right)
$$

Os dados obtidos foram avaliados em delineamento inteiramente casualizado pelo método de análise de variância (ANOVA), foi aplicado o teste de Tukey a 5\% probabilidade. A análise estatística foi realizada utilizando programa ASSISTAT (versão 7,7).

\section{Resultados e Discussão}

A Tabela 3 demonstra as análises físicas e químicas realizadas nas três formulações da geleia de graviola com pimenta tipo comum e extra.

Em relação ao $\mathrm{pH}$, a geleia comum não apresentou diferença significativa entre as amostras com 0,5 e 1,5 $\%$ de pectina e entre 1,0 e $1,5 \%$ de pectina, entretanto, na geleia extra não houve diferença significativa entre nenhuma das concentrações de pectina estudadas e nas concentrações de 1,0 e $1,5 \%$ da geleia extra não apresentou diferença entre as amostras das geleias 
comum com 0,5 e $1,5 \%$ de pectina. $\mathrm{O}$ índice de $\mathrm{pH}$ apresenta-se levemente acima do recomendado que seria de 3,4, sendo que, abaixo de 3,0, ocorre uma tendência a sinérese. Os valores encontrados para as formulações de geleia foram semelhantes aos encontrados por Freitas et al. (2008), em avaliação de geleia de gabiroba que obteve valores médios de 3,87 .

A análise de acidez demonstrou que na geleia comum apenas as amostras com $0,5 \%$ de pectina diferiram das demais concentrações, enquanto que na geleia extra não houve diferença significativa entre as concentrações. Segundo Torrezan (1998), o ácido enrijece as fibras da rede, mas a alta acidez afeta a elasticidade, devido à hidrólise da pectina. A acidez total da geleia deve estar ao redor de $0,5-0,8 \%$, pois, acima de $1 \%$ ou abaixo de $0,5 \%$, tende a ocorrer sinérese, ou seja, exsudação do líquido da geleia. Portanto, na amostra comum com maior valor de acidez encontrado a $0,5 \%$ de pectina foi a única a apresentar tendência a sinérese.

Como foi possível observar, todas as formulações de geleias do tipo comum atingiram a concentração de 65 ${ }^{\circ}$ Brix, similares aos valores médios obtidos por Araújo et al. (2012) em geleia de pimenta com abacaxi, a qual também obteve valores de $65^{\circ}$ Brix. Para a geleia do tipo extra, apenas as amostras com $1,0 \%$ de pectina apresentaram diferença significativa em relação às demais concentrações de pectina e na geleia comum não apresentaram diferença significativa; resultado semelhante ao encontrado por Pereira et al. (2011) em geleia de marmelo japonês.

Pode-se observar que as formulações de geleia comum obtiveram maior teor de atividade de água; fato não esperado visto que as formulações têm maior quantidade de açúcar e tendem a absorver mais umidade. As formulações de geleia extra e comum foram estatisticamente significativas entre as concentrações de pectinas avaliadas quanto ao teor de água presente nas amostras. A média estimada foi de 0,81 , todavia foram estatisticamente superiores às amostras do tipo extra cujo valor menos expressivo da atividade de água ficou em torno de 0,72 quando utilizado $1,5 \%$ de pectina.

Em relação ao teor de umidade não houve diferença estatística significativa entre as concentrações de pectina e entre os tipos de geleia. Os valores de umidade obtidos foram semelhantes aos encontrados por Freitas (2008), que encontraram valores médios de 34,33 $\mathrm{g} / 100 \mathrm{~g}$ para geleia de gabiroba.

A análise de vitamina $\mathrm{C}$ em todas as amostras de geleia comum e extra obtiveram como resultado 0,889 $\mathrm{g} / 100 \mathrm{~g}$ de ácido ascórbico, sendo que Schervensquy et al. (2015) encontraram valor inferior para geleia de abacaxi com hortelã que foi de $0,52 \mathrm{mg} / 100 \mathrm{~g}$ de ácido ascórbico.

$\mathrm{Na}$ Tabela 4 pode-se verificar os valores obtidos nas análises de cor $\left(\mathrm{L}^{*}, \mathrm{a}^{*}, \mathrm{~b}^{*}\right)$ das geleias de graviola com pimenta dedo-de-moça extra e comum.

Tabela 3. Análises físicas e químicas das formulações de geleia de graviola comum e extra com adição de pimenta dedo-de-moça com $0,5 \%, 1,0 \%$, e $1,5 \%$ de pectina.

\begin{tabular}{|c|c|c|c|c|c|c|}
\hline \multirow[b]{2}{*}{ Análises } & \multicolumn{3}{|c|}{ Comum } & \multicolumn{3}{|c|}{ Extra } \\
\hline & $\begin{array}{c}\text { Pectina } \\
0,5 \%\end{array}$ & $\begin{array}{c}\text { Pectina } \\
1,0 \%\end{array}$ & $\begin{array}{c}\text { Pectina } \\
1,5 \%\end{array}$ & $\begin{array}{c}\text { Pectina } \\
0,5 \%\end{array}$ & $\begin{array}{c}\text { Pectina } \\
1,0 \%\end{array}$ & $\begin{array}{c}\text { Pectina } \\
1,5 \%\end{array}$ \\
\hline $\mathrm{pH}$ & $\begin{array}{c}3,73 \\
\pm 0,057^{\mathrm{BC}}\end{array}$ & $\begin{aligned} & 3,93 \\
\pm & 0,057^{\mathrm{A}}\end{aligned}$ & $\begin{array}{c}3,83 \\
\pm 0,057^{\mathrm{AB}}\end{array}$ & $\begin{array}{l}3,69 \\
\pm 0,057^{\mathrm{C}}\end{array}$ & $\begin{array}{c}3,76 \\
\pm 0,057^{\mathrm{BC}}\end{array}$ & $\begin{array}{c}3,77 \\
\pm 0,057^{\mathrm{BC}}\end{array}$ \\
\hline $\begin{array}{l}\text { Acidez(\% de ácido } \\
\text { cítrico) }\end{array}$ & $\begin{array}{c}2,25 \\
\pm 0,00^{\mathrm{A}}\end{array}$ & $\begin{aligned} & 1,25 \\
\pm & 0,00^{\mathrm{BC}}\end{aligned}$ & $\begin{array}{l}1,0 \\
\pm 0,00^{\mathrm{C}}\end{array}$ & $\begin{aligned} & 1,65 \\
\pm & 0,00^{\mathrm{B}}\end{aligned}$ & $\begin{array}{c}1,65 \\
\pm 0,00^{\mathrm{B}}\end{array}$ & $\begin{array}{c}1,65 \\
\pm 0,00^{\mathrm{B}}\end{array}$ \\
\hline $\operatorname{SST}\left({ }^{\circ}\right.$ Brix $)$ & $\begin{array}{c}65,0 \\
\pm 0,00^{\mathrm{A}}\end{array}$ & $\begin{array}{c}65,0 \\
\pm 0,00^{\mathrm{A}}\end{array}$ & $\begin{array}{c}65,0 \\
\pm 0,00^{\mathrm{A}}\end{array}$ & $\begin{array}{c}65,0 \\
\pm 0,00^{\mathrm{A}}\end{array}$ & $\begin{array}{c}67,0 \\
\pm 0,00^{\mathrm{B}}\end{array}$ & $\begin{array}{c}65,0 \\
\pm 0,00^{\mathrm{A}}\end{array}$ \\
\hline Aw & $\begin{array}{c}0,81 \\
\pm 0,003^{\mathrm{A}}\end{array}$ & $\begin{array}{c}0,81 \\
\pm 0,004^{\mathrm{A}}\end{array}$ & $\begin{aligned} & 0,80 \\
\pm & 0,001^{\mathrm{A}}\end{aligned}$ & $\begin{array}{c}0,75 \\
\pm 0,01^{\mathrm{B}}\end{array}$ & $\begin{array}{c}0,75 \\
\pm 0,01^{\mathrm{B}}\end{array}$ & $\begin{array}{c}0,72 \\
\pm 0,004^{\mathrm{C}}\end{array}$ \\
\hline Umidade (\%b.u) & $\begin{array}{c}34,64 \\
\pm 1,38^{\mathrm{A}}\end{array}$ & $\begin{array}{c}30,27 \\
\pm 5,66^{\mathrm{A}}\end{array}$ & $\begin{array}{c}38,87 \\
\pm 1,23^{\mathrm{A}}\end{array}$ & $\begin{array}{c}34,53 \\
\pm 1,42^{\mathrm{A}}\end{array}$ & $\begin{array}{r}28,72 \\
\pm 4,52^{\mathrm{A}}\end{array}$ & $\begin{array}{c}37,45 \\
\pm 2,05^{\mathrm{A}}\end{array}$ \\
\hline $\begin{array}{l}\text { Vitamina C } \\
(\mathrm{mg} / 100 \mathrm{~g})\end{array}$ & $0,889^{\mathrm{A}}$ & $0,889^{\mathrm{A}}$ & $0,889^{\mathrm{A}}$ & $0,889^{\mathrm{A}}$ & $0,889^{\mathrm{A}}$ & $0,889^{\mathrm{A}}$ \\
\hline Sinerése $(\%)$ & 0,0014 & -- & - & - & - & -- \\
\hline
\end{tabular}

*As letras maiúscula diferentes em uma mesma linha, apresentam diferença estatística entre si pelo teste de Tukey (p <0,05). 
Em relação ao parâmetro $L^{*}$ (luminosidade ou claridade) houve variações de 21,59 a 27,25, sendo a menor luminosidade para a geleia extra a $0,5 \%$ de pectina e maior luminosidade na geleia extra a $1,0 \%$ de pectina, observando-se que o tipo de geleia e o teor de pectina pode ter influenciado na luminosidade.

Para o parâmetro $\mathrm{a}^{*}$ [coloração vermelha(+) ao verde(-)], as amostras de $0,5 \%$ de pectina da geleia extra apresentaram dados estatisticamente iguais às amostras com $1,5 \%$ da geleia comum, no entanto, a geleia extra a $1,5 \%$ de pectina apresentou maior tendência à coloração vermelha.

$\mathrm{O}$ parâmetro de cor $\mathrm{b} *($ coloração $\operatorname{amarelo(+)}$ ao azul(-), apresentou diferenças significativas entre os tipos de geleias e as concentrações de pectinas avaliados. De modo geral os valores oscilaram entre 15,60 na geleia comum a 25,28 na geleia extra na concentração de $0,5 \%$ de pectina. As amostras de geleia extra com 0,5 e $1,5 \%$ de pectina a apresentaram maiores valores para o parâmetro $b$ *indicando uma tendência de coloração amarela.

O valor de croma encontrado variou de 15,84 a 25,94 e para o ângulo da e hue oscilou entre 1,26 e 1,39. $\mathrm{O}$ valor de $\mathrm{c}^{*}$ foi menor nas geleias do tipo comum, exceção a $1 \%$ de pectina nas amostras do tipo extra.

De acordo com Kirca et al. (2007) a cor perceptível de geleias depende da quantidade relativa de cores vermelha e amarela, a qual é expressa como $\mathrm{h}^{*}$, enquanto que o valor de $\mathrm{c}^{*}$ descreve a saturação ou a intensidade da cor em que as geleias com maiores valores de $\mathrm{c}^{*}$ são mais vivas e, consequentemente, mais atrativas à compra. No presente estudo as formulações de geleias de graviola que apresentaram uma coloração $\mathrm{c}^{*}$ acentuada e com brilho característico foram as $0,5 \mathrm{e}$ $1,5 \%$ geleia extra.

Tabela 4. Análises de cor realizadas nas formulações de geleia de graviola comum e extra com adição de pimenta, com $0,5 \%, 1,0 \%$, e $1,5 \%$ de pectina

\begin{tabular}{|c|c|c|c|c|c|c|}
\hline \multirow[b]{2}{*}{ Tratamentos } & \multicolumn{2}{|c|}{$\mathrm{L}^{*}$} & \multicolumn{2}{|c|}{$a^{*}$} & \multicolumn{2}{|c|}{$\mathrm{b}^{*}$} \\
\hline & Extra & Comum & Extra & Comum & Extra & Comum \\
\hline $5 \%$ & $\begin{array}{c}21,59 \\
\pm 0,02^{\mathrm{a}}\end{array}$ & $\begin{array}{c}26,73 \\
\pm 0,01^{b}\end{array}$ & $\begin{array}{c}5,83 \\
\pm 0,01^{\mathrm{a}}\end{array}$ & $\begin{array}{c}2,71 \\
\pm 0,02^{b}\end{array}$ & $\begin{array}{c}25,28 \\
\pm 0,08^{\mathrm{a}}\end{array}$ & $\begin{array}{c}15,60 \\
\pm 0,05^{\mathrm{b}}\end{array}$ \\
\hline $1,0 \%$ & $\begin{array}{c}27,25 \\
\pm 0,04^{b}\end{array}$ & $\begin{array}{c}24,98 \\
\pm 0,01^{c}\end{array}$ & $\begin{array}{c}3,50 \\
\pm 0,03^{\mathrm{b}}\end{array}$ & $\begin{array}{c}3,88 \\
\pm 0,02^{\mathrm{c}}\end{array}$ & $\begin{array}{c}18,87 \\
\pm 0,03^{\mathrm{b}}\end{array}$ & $\begin{array}{r}19,33 \\
\pm 0,03^{\mathrm{c}}\end{array}$ \\
\hline $1,5 \%$ & $\begin{array}{c}24,42 \\
\pm 0,03^{\mathrm{c}}\end{array}$ & $\begin{array}{c}24,21 \\
\pm 0,016^{d}\end{array}$ & $\begin{array}{c}6,20 \\
\pm 0,05^{\mathrm{c}}\end{array}$ & $\begin{array}{c}5,81 \\
\pm 0,10^{\mathrm{d}}\end{array}$ & $\begin{array}{c}23,51 \\
\pm 0,05^{\mathrm{c}}\end{array}$ & $\begin{array}{c}18,10 \\
\pm 0,28^{\mathrm{d}}\end{array}$ \\
\hline
\end{tabular}

*As letras minúsculas diferentes horizontalmente ou verticalmente, dentro de um mesmo espaço de cor, apresentam diferença estatística entre si pelo teste de Tukey ( $<<0,05), L^{*}: \% C V=0,29 ; a^{*}: C V=1,0 \% ; b^{*}: C V=0,61 \%$

Tabela 5. Análises da tonalidade croma e hue nas formulações de geleia de graviola comum e extra com adição de pimenta, com $0,5 \%, 1,0 \%$, e $1,5 \%$ de pectina.

\begin{tabular}{ccccc}
\hline Tratamentos & \multicolumn{2}{c}{ CROMA $\left(\mathrm{c}^{*}\right)$} & \multicolumn{2}{c}{ HUE $\left(\mathrm{h}^{*}\right)$} \\
\hline & Extra & Comum & Extra & Comum \\
\cline { 2 - 5 } $0,5 \%$ & $25,94 \mathrm{c}$ & $15,84 \mathrm{a}$ & $1,34 \mathrm{~b}$ & $1,39 \mathrm{c}$ \\
$1,0 \%$ & $19,19 \mathrm{~b}$ & $19,71 \mathrm{~b}$ & $1,38 \mathrm{c}$ & $1,37 \mathrm{c}$ \\
$1,5 \%$ & $24,13 \mathrm{c}$ & $19,01 \mathrm{~b}$ & $1,31 \mathrm{~b}$ & $1,26 \mathrm{a}$ \\
\hline
\end{tabular}

*As letras minúsculas diferentes horizontalmente ou verticalmente, dentro de um mesmo espaço de cor, apresentam diferença estatística entre si pelo teste de Tukey $(\mathrm{p}<0,05)$.

\section{Conclusões}

Pelos resultados encontrados neste trabalho ficou evidente que a concentração de pectina e o tipo de geleia influi nas características físicas e químicas da geleia de graviola com pimenta dedo-de-moça. A geleia comum com $5 \%$ de pectina apresentou sinérese, alteração indesejável; as geleias extras apresentaram uma menor atividade de água o que evidencia uma maior shelf life.

O processamento de geleias é um modo de aproveitamento de frutos sazonais. Apesar da graviola ser uma fruta de grande valor nutricional ainda são escassos estudos que viabilizem seu melhor aproveitamento. 


\section{Referências Bibliográficas}

ARAÚJO, E. R.; RÊGO, E. R.; SAPUCAY, M. J. C.; RÊGO, M. M.; SANTOS, R. M. C. Elaboração e análise sensorial de geleia de pimenta com abacaxi. Revista Brasileira de Produtos Agroindustriais, Campina Grande-PB, v.14, n.3, p.233-238, 2012.

BRASIL/MS. -Ministério da Saúde. ANVISA-AGÊNCIA NACIONAL DE VIGILÂNCIA SANITÁRIA. Resolução CNNPA - Comissão Nacional de Normas e Padrões Para Alimentos $\mathrm{n}^{\circ} 12$, de 1978. Diário Oficial da República Federativa do Brasil, Brasília, DF, 24 jul. 1978.

CARVAlHO, S. I. C.; BIANCHETTI; L. B. Sistema de Produção de Pimentas (Capsicumspp.): Botânica. Embrapa Hortaliças, Sistemas de Produção, 2004.

FREITAS, J. B.; CÂNDIDO, T. L. N.; SILVA, M. R. Geleia de gabiroba: avaliação da aceitabilidade e características físicas e químicas. Pesquisa Agropecuária Tropical. Goiânia GO, v. 38, n. 2, p. 87-94, 2008.

IAL.INSTITUTO ADOLFO LUTZ. Métodos físico-químicos para analise de alimentos. 4.ed., 1.ed. Instituto Adolfo Lutz, São Paulo - SP, 2008.

KIRCA, A.; ÖZKAN, M.; CEMEROĞLU, B. Storage stability of strawberry jam color enhanced with black carrot juice concentrate. Journal of Food Processing and Preservation, Turkey, v. 31, p. 531-545, 2007.
LICODIEDOFF, S.; AQUINO, A. D.; GODOY, R. C. B.; LEDO, C. A. S. Avaliação da sinérese em geleia de abacaxi por meio de análise uni e multivariada. Semina: Ciências Exatas e Tecnológicas, Londrina-PR, v. 31, n. 1, p. 51-56, 2010.

PEREIRA, G. G., ALVARENGA, A. A., ABRAHÃO, E. PINHEIRO, A. C. M., OLIVEIRA, A. F., PIO. R. Avaliação sensorial de geleia de marmelo 'Japonês' em diferentes concentrações de sólidos solúveis totais. Brazilian Journal of Food Technology, Campinas- SP, v. 14, n. 3, p. 226-231, 2011.

SACRAMENTO, C. K. Caracterização física e química de frutos de três tipos de gravioleira (Annonamuricata L.). Revista Brasileira de Fruticultura, Jaboticabal-SP, v. 25, n. 2, p. 329-331, 2003.

SCHERVENSQUY, E. M. JOCIÉLI EURICH, J.; JESUS, M. A. T.; LORENE SIMIONI YASSIN, L. S.; BORSATO, A. V.; RAUPP, D. S. Desenvolvimento de geleia light de abacaxi com hortelã. Journal Health, Campos Gerais-MG, $13^{\text {a }}$ ed., 2015.

TORREZAN, R. Manual para a produção de geleias de frutas em escala industrial. Rio de Janeiro-RJ: EMBRAPA/CTAA, 1998. 27 p. 